\title{
Participación en actividades fuera de la escuela y su valor subjetivo: un estudio exploratorio con niños y adolescentes
}

\section{Judith Oller ${ }^{1}$, Mariana Largo ${ }^{1}$, Iris Merino ${ }^{1}$, César Coll ${ }^{1}$}

${ }^{1}$ Departamento de Cognición, Desarrollo y Psicología de la Educación. Universitat de Barcelona

\section{España}

Correspondencia: Judith Oller. Departamento de Cognición, Desarrollo y Psicología de la Educación. Universitat de Barcelona. E-mail: jollerb@ub.edu 


\section{Resumen}

Introducción. En el marco de la "nueva ecología del aprendizaje” ha empezado a ser fundamental trazar puentes entre las experiencias de aprendizaje que los estudiantes desarrollan dentro y fuera de la escuela. Hasta el momento, pocas investigaciones se han enfocado en el estudio de actividades que tienen lugar fuera de la escuela. En este trabajo exploramos las diferencias en la participación de niños y adolescentes en 26 actividades fuera de la escuela, y la percepción subjetiva de los aprendizajes derivados de su participación en ellas.

Método. Diseñamos un cuestionario para explorar la participación de estudiantes en actividades fuera de la escuela y el valor subjetivo que atribuyen a sus experiencias de aprendizaje. La lista de actividades se obtuvo a través del análisis temático de 17 grupos focales. La muestra son 946 niños de entre 10 y 16 años de escuelas públicas de Cataluña. La participación se analiza en función de la edad, el sexo, el nivel socioeconómico y la localización geográfica de la escuela. El análisis de los datos se realiza con el software SPSS.

Resultados. Se evidencian diferentes tendencias en la participación en actividades fuera de la escuela según la edad y el sexo de los participantes, mientras que factores como el nivel socioeconómico y la localización geográfica tienen una influencia muy limitada. La participación en actividades fuera de la escuela muestra un patrón de desarrollo en función de la edad, así como diferencias importantes relacionadas con el género (los chicos están sobrerrepresentados en "deportes" y "videojuegos", mientras que las chicas muestran una mayor preferencia por actividades sociales o académicas). Los participantes califican las actividades relacionadas con lo académico como las más importantes a nivel de aprendizaje en contraste con otros tipos de actividades informales en las que dicen que también aprenden.

Discusión y Conclusiones. Consideramos necesario que las escuelas sean conscientes del riesgo que pueden tener los estereotipos de género y los discursos sociales sobre el aprendizaje formal en la toma de decisiones de los estudiantes. Se deben implementar nuevas formas de validar las experiencias de aprendizaje no-formales e informales para reconocer y aumentar la visibilidad social de otras experiencias formativas fuera de la escuela.

Palabras clave: actividades de aprendizaje, actividades fuera de la escuela, características sociodemográficas, experiencias de aprendizaje 


\section{Abstract}

Introduction. In the new ecology of learning it is becoming crucial to trace paths among the learning experiences that students develop inside and outside the school. Few research studies have focused on out-of-school activities. In this paper, we explore children and adolescents 'differences in participation in 26 out-of-school activities, and their subjective perception of learning derived from its own participation.

Method. We designed a questionnaire to explore the participation in out-of-school activities and the subjective value that students attribute to their learning experiences. The list of activities was obtained through thematic analysis of 17 previous focus groups. Participants are 946 children, aged either 10 or 16, from public schools in Catalonia. Participation is analysed according to age, gender, SES, and students' area of residence. Data analysis is performed with SPSS software.

Results. Different trends in the participation in out-of-school activities appear according to participants' age and gender, whereas factors such as SES and area of residence do have a very limited influence. Engagement in out-of-school activities show a developmental pattern according to participants' age, as well as important gender-related differences (boys are overrepresented in "sports" and "playing video games", while girls show a greater preference for choosing social- or academic-oriented activities). The whole sample rate academic-related activities as more important than other kinds of informal activities in which they also learn.

Conclusions and Discussion. We find necessary that schools be aware of the risk that gender stereotypes and social discourses about formal learning can have in the decision making of students. New ways of validating non-formal and informal learning experiences should be implemented to recognize and increase the social visibility of other formative experiences developed outside formal contexts.

Keywords: learning activities, out-of-school activities, sociodemographic features, learning experiences 


\section{Introducción}

\section{La nueva ecología del aprendizaje}

En la actualidad, la Sociedad de la Información (SI) plantea retos a los sistemas educativos debido a la emergencia de nuevas y múltiples formas de participación en actividades educativas, profesionales y de ocio. Mucho más que antes, las competencias de los estudiantes en la SI dependen de los diferentes contextos por los que transitan y que les ofrecen oportunidades para aprender más allá del contexto escolar. Algunos de estos contextos informales y no-formales pueden ofrecer oportunidades de aprendizaje ricas para los estudiantes, mientras que otros brindan menos oportunidades o incluso podríamos decir que son escenario de experiencias de aprendizaje inadecuadas para cumplir con las necesidades personales, educativas y profesionales de los estudiantes. Por lo tanto, el riesgo de que las desigualdades económicas y culturales se transformen en desigualdades educativas se vuelve más agudo (Holland y Lave, 2009).

Barron (2006) acuñó el concepto de "ecología del aprendizaje" para describir el conjunto de contextos interconectados, físicos o virtuales, que proveen a las personas oportunidades y recursos para continuar aprendiendo a lo largo de la vida. En esta línea, es fundamental que los sistemas educativos conciban el aprendizaje como un proceso que se desarrolla tanto a lo largo como a lo ancho de la vida, pues también permite reconocer el carácter dinámico de las necesidades educativas de las personas en el contexto social y posibilita el logro de los objetivos personales futuros (Banks et al., 2007).

Coll (2018) plantea que estamos ante una "nueva ecología del aprendizaje", donde el aprendizaje es entendido como una trayectoria individual conformada por diferentes experiencias subjetivas de aprendizaje en contextos específicos y que, en muchas ocasiones, dicho aprendizaje puede ser expandido a otros contextos y momentos. Las personas aprendemos participando en actividades que tienen una clara intencionalidad educativa, pero también en otras que no tienen esa intencionalidad. En este sentido, hay un número creciente de alumnos para los que las actividades que se realizan dentro de la escuela tienen poco sentido (a pesar de su elevada intencionalidad educativa) y, por tanto, sus aprendizajes en este contexto están lejos de ser significativos (Coll, 2016). Por lo contrario, este alumnado suele encontrar fuera de la escuela actividades y oportunidades de aprender más relevantes y 
significativas para ellos, que responden mejor a sus intereses personales y necesidades de aprender.

En este sentido, entendemos las trayectorias de aprendizaje como un conjunto de experiencias de aprendizaje interrelacionadas que las personas dibujan a partir de su participación en actividades situadas en diferentes contextos de actividad y a lo largo de su vida. Desde nuestra aproximación, es esencial el estudio de las trayectorias individuales de aprendizaje y de los factores que influyen en su configuración, pues dicha exploración nos ayudaría a garantizar las mismas oportunidades educativas independientemente de la clase social, edad, cultura o género del aprendiz, así como a repensar la educación formal situada en un modelo de educación distribuida e interconectada (Coll, 2013). En concordancia con otros estudios centrados en el contexto de aprendizaje (e.g. Erstad et al., 2016), hemos transitado desde una mirada centrada en las características de los contextos de aprendizaje hacia otra enfocada en las experiencias de aprendizaje que tienen lugar en contextos de actividad, y también en la comprensión respecto a cómo todas las experiencias de aprendizaje pueden llegar a estar interrelacionadas (Engel et al., 2019).

Considerando estas ideas, uno de los retos que enfrentan las instituciones educativas es definir "cómo" y "con qué finalidad" desde las escuelas se pueden establecer relaciones entre los diferentes contextos a fin de facilitar que los estudiantes conecten sus experiencias de aprendizaje, promover en ellos un mayor sentido de los aprendizajes escolares y procurar la equidad educativa para todo el alumnado (Bronkhorst y Akkerman, 2016; Coll, 2013; Leander, Philips, y Taylor, 2010; Sefton-Green, 2012). Ante este complejo reto, resulta relevante conocer en profundidad cuáles son los factores más influyentes que afectan las trayectorias de aprendizaje de las personas, así como cuál es el rol de esos factores en sus experiencias de aprendizaje (DiGiacomo et al., 2018).

Características sociodemográficas que afectan la participación en actividades fuera de la escuela

Actualmente, hay un número considerable de investigaciones que demuestran que las actividades fuera de la escuela en las que participan los jóvenes afectan significativamente a su desarrollo social, educativo, cívico y físico (Feldman y Matjasko, 2005; Shulruf, 2010). En general, los estudios que abordan este tópico están enfocados en la participación de niños y jóvenes en actividades extracurriculares, es decir, en su participación en actividades 
estructuradas que siguen un formato similar al de la escuela, pero que se llevan a cabo en contextos fuera de esta. Para algunos autores, como Baber et al. (2009), el interés en este tipo de actividades se centra en el rol que juegan en la prevención de conductas de riesgo, abandono escolar y rendimiento académico exitoso. Desde nuestro punto de vista, las actividades fuera de la escuela no son solo las actividades extracurriculares, sino que abarcan todas aquellas que no se lleven a cabo en el contexto educativo formal, independientemente del grado de formalidad e institucionalización, incluyéndose, como consecuencia, una gran cantidad y variedad de actividades que los niños y adolescentes realizan más allá de la escuela y en las que pueden o no aprender (Trilla et al., 2003). Nuestro interés por las actividades fuera del contexto escolar se da por el potencial que ofrecen para generar experiencias de aprendizaje ricas y diversas que promuevan el aprendizaje a lo largo y ancho de la vida.

El aspecto crítico respecto al acceso y diversidad de contextos en los que participan los jóvenes es que, mientras algunos de ellos tendrán disponibles múltiples oportunidades, recursos y apoyo social para participar en una gran diversidad de actividades fuera de la escuela, otros adolescentes vivirán restricciones para acceder a este tipo de actividades debido a la confluencia de diversos factores (Lareau, 2011). En este sentido, y tal como lo plantean Philip y Azevedo (2017), qué y cómo aprenden las personas hoy en día está lejos de ser algo neutral, por lo que es crucial considerar las dimensiones socioeconómicas y sociopolíticas del aprendizaje (es decir, las relaciones asimétricas de poder y el posicionamiento de algunos programas de actividades extraescolares con relación a otros, las concepciones industriales de productividad en la era de la educación guiada por el mercado...). En este escenario, la selección de actividades fuera de la escuela está influida por una combinación de factores personales y contextuales (Bouffard et al., 2006), siendo las características sociodemográficas los predictores más comunes de participación. De acuerdo con Mahoney y colaboradores (2009), aspectos como la edad, el nivel socioeconómico, el género y la raza o etnia pueden afectar las posibilidades de acceso y el tipo de actividades disponibles para los jóvenes. Del mismo modo, las competencias, intereses y motivaciones individuales son elementos centrales de la participación (Sefton-Green, 2012). Finalmente, estos factores demográficos y personales están respaldados por los contextos inmediatos en los que se desarrollan los individuos, incluidos los grupos de amigos, las familias, las escuelas, los vecindarios y las actividades mismas. En general, todas las variables mencionadas pueden ser generadoras potenciales de desigualdades, ya que tienen un impacto en la selección de las actividades y de 
los contextos en los que los jóvenes participan y, por lo tanto, en la cantidad y la calidad de las experiencias de aprendizaje posibles.

En relación con la variable edad, los estudios señalan que la participación de los niños en las actividades escolares generalmente aumenta a lo largo de la etapa de educación primaria y alcanza su punto máximo en la adolescencia (Simpkins et al., 2005). El momento de mayor participación en ciertas actividades de acuerdo con la edad depende, en parte, del tipo de actividad. Por ejemplo, se ha observado que la participación en actividades deportivas y de artes escénicas alcanza su punto máximo en primaria, mientras que la participación en el gobierno, u otros clubes escolares, parece alcanzar su punto máximo en secundaria (Darling, 2005). Estos cambios debido a la edad surgen por varias razones. En primer lugar, y en comparación con la infancia, los adolescentes necesitan pasar una cantidad de tiempo considerable haciendo deberes o contribuyendo a la familia a través de las tareas domésticas. En segundo lugar, es probable que los adolescentes pasen su tiempo con amigos o sin supervisión debido, en parte, a un incremento en su autonomía. En tercer lugar, los adolescentes pueden escoger más actividades que los niños pequeños porque, además de la escuela, pueden participar en actividades comunitarias (Mahoney et al., 2009).

Otro hallazgo relevante es que los niños y adolescentes que pertenecen a familias de nivel socioeconómico alto tienen más probabilidades de participar en actividades extraescolares organizadas que los adolescentes de familias de nivel socioeconómico bajo (e.g. Kadar-Satat, 2014; Simpkins et al., 2005). En este sentido, son significativas y preocupantes las barreras que existen en la participación de los adolescentes que provienen de familias con bajos ingresos en este tipo de actividades.

La influencia significativa de la variable sexo se ha demostrado tanto en actividades extracurriculares (Eccles et al., 2003; Feldman y Matjasko, 2005) como en actividades de ocio (Bruyn y Cillessen, 2008), señalando la desigualdad de acceso entre hombres y mujeres a ciertos campos del conocimiento y entornos profesionales, especialmente los relacionados con la ciencia, la tecnología, la ingeniería y las matemáticas (Davaki, 2016; Engel et al., 2018). Bruyn y Cillessen (2008) examinaron la participación de jóvenes en setenta actividades fuera de la escuela y, según los resultados, agruparon las actividades en cuatro tipos: las actividades sociales; las actividades creativas; las actividades relacionadas con deportes y automóviles; y las actividades que incluían el uso de ordenadores. Sus resultados indicaron que las chicas 
participan más en los dos primeros tipos de actividades, mientras que los chicos lo hacen más en los dos últimos tipos.

En este orden de ideas, y teniendo en cuenta los resultados a los que apuntan las investigaciones en torno al sexo, asumimos que tanto niños como adolescentes construyen significados sobre los estereotipos de género durante su participación en actividades de aprendizaje dentro de sus contextos cotidianos de socialización, ya que es en esos contextos donde se construyen formas específicas de concebir, interpretar y actuar sobre la realidad. Los estereotipos de género, definidos como las representaciones culturales de los atributos y el papel de mujeres y hombres en diversos escenarios sociales (Colás y Villaciervos, 2007), influyen en la selección y participación en determinadas actividades, junto con otros factores mencionados previamente, como el nivel socioeconómico, los antecedentes familiares y el origen (Eccles, 2014).

En España, algunos estudios han señalado diferencias en la participación de chicas y chicos jóvenes en diversas áreas de actividad. Respecto a las actividades deportivas, en el trabajo de Nuviala y colaboradores (2009) se detecta que las chicas entre 12 y 16 años realizan menos actividades físicas que los chicos. Mosquera y Puig (2002) sugieren que los valores y las normas adquiridos durante el proceso de socialización son importantes para explicar esta diferencia, ya que los deportes están asociados con valores que tradicionalmente se consideran masculinos (es decir, competir para ser el mejor, la fuerza y el poder, el éxito, etc.). Por estas razones, a muchas chicas que no han sido educadas para "practicar un deporte", este tipo de actividades no les parece una propuesta atractiva.

En cuanto a la participación de niños en actividades extraescolares, Molinuevo (2009) evaluó las diferencias de participación de 273 estudiantes de segundo, cuarto y sexto de primaria en Cataluña según su género y edad. El estudio encontró que los chicos jugaban a videojuegos con mucha más frecuencia que las chicas (78\% frente a 36\%), mientras que las chicas se dedicaban significativamente más a la lectura que los chicos (89\% frente a $83 \%)$. Molinuevo (2009) también señala que, en general, los chicos pasan más tiempo mirando la televisión, participando en actividades extraescolares como deportes y videojuegos, mientras que las chicas pasan más tiempo haciendo deberes, leyendo, estudiando idiomas o haciendo música y danza. 
En resumen, en las últimas décadas ha habido un interés creciente por estudiar la participación de niños y adolescentes en actividades fuera de la escuela. Sin embargo, la mayoría de ellos se han centrado en las actividades extraescolares dirigidas, dejando de lado actividades informales y/o actividades que puedan llevarse a cabo en distintos contextos. De igual modo, no hay estudios que analicen los motivos personales de los jóvenes para elegir las actividades que realizan fuera de la escuela, así como la importancia que atribuyen a su participación en esas actividades y los aprendizajes que realizan en ellas.

Impulsados por el deseo de desarrollar y ampliar la investigación en el campo del aprendizaje y el desarrollo, y en el marco de la nueva ecología del aprendizaje (Barron, 2006; Coll, 2013), hemos diseñado un estudio exploratorio que busca indagar las diferencias en la participación de los niños y jóvenes en actividades fuera de la escuela en función de cuatro variables sociodemográficas que los trabajos previamente revisados relacionan con mayores o menores oportunidades de acceso a actividades fuera de la escuela con potencial para el aprendizaje. La elección de estos criterios responde, por tanto, a la conjetura de que las cuatro variables implicadas tienen una incidencia en la variedad y características de los contextos de actividad en los que participan los niños y adolescentes, en las oportunidades y recursos para aprender que encuentran en esos contextos, en las experiencias de aprendizaje que en ellos pueden obtener y, en definitiva, en la configuración de sus trayectorias individuales de aprendizaje.

\section{Objetivos}

El presente estudio forma parte de una investigación más amplia cuya finalidad es estudiar las experiencias subjetivas de aprendizaje que conforman las trayectorias individuales de aprendizaje de niños y adolescentes en edad escolar. En este marco, el estudio persigue dos objetivos:

- Describir las diferencias en la participación de niños y adolescentes en actividades fuera de la escuela atendiendo a factores como la edad, el género, el nivel socioeconómico y la localización geográfica.

- Analizar el valor subjetivo que los niños y adolescentes otorgan a los aprendizajes realizados en actividades fuera de la escuela, su importancia y los motivos para participar en ellas. 


\section{Método}

Este estudio exploratorio forma parte de la segunda fase de un proyecto de investigación más amplio que tiene un enfoque multimétodo en el que se combinan instrumentos y procedimientos de recogida y análisis de datos de carácter cuantitativo y cualitativo. El proyecto se ha desarrollado en tres fases y los resultados de cada una constituyen el punto de partida de la siguiente. En la primera fase se identificaron, por medio de grupos focales, las actividades en las que participan habitualmente los niños y jóvenes fuera del contexto escolar. La segunda fase consistía en explorar la participación de niños y jóvenes en las actividades identificadas en la fase 1 y las características de los contextos socio-institucionales en los que llevan a cabo estas actividades mediante un cuestionario. La finalidad de la tercera fase fue explorar en profundidad las experiencias subjetivas de aprendizaje relatadas por una muestra de niños y jóvenes en entrevistas semiestructuradas.

\section{Participantes}

El estudio se ha llevado a cabo con una muestra de 946 estudiantes pertenecientes a 22 centros educativos públicos de diferentes municipios de las cuatro provincias de Cataluña. La unidad de muestreo fueron los centros educativos, que se seleccionaron intencionalmente asegurando que estuvieran presentes, de la manera más equilibrada posible, las diferentes combinaciones de los tres criterios siguientes: la etapa educativa (escuelas de educación primaria e institutos de educación secundaria), la localización geográfica de la escuela (zonas urbanas: municipios con más de 10.000 habitantes; y rurales: municipios con menos de 10.000 habitantes), y nivel socioeconómico (nivel alto o medio-alto, y nivel bajo o medio-bajo). De cada escuela, los participantes fueron seleccionados en función de la edad y el curso escolar: niños de 10 años (más/menos 6 meses), es decir, alumnado de cuarto curso de educación primaria y jóvenes de 16 años (más/menos 6 meses) que cursaban cuarto de educación secundaria obligatoria.

En la Tabla 1 se describen las características de los participantes en función de la localización geográfica de la escuela, el nivel socioeconómico, el género y la edad. 
Tabla 1. Características de la muestra

\begin{tabular}{llll}
\hline & & $N$ & $\%$ \\
\hline $\begin{array}{llll}\text { Localización } \\
\text { geográfica }\end{array}$ & Rural & 434 & 45,9 \\
& Urbana & 512 & 54,1 \\
\hline Nivel SE & Bajo & 592 & 62,6 \\
& Alto & 354 & 37,4 \\
\hline Género & Femenino & 498 & 52,6 \\
& Masculino & 448 & 47,4 \\
\hline Edad & 10 años & 411 & 43,4 \\
& 16 años & 535 & 56,5 \\
\hline
\end{tabular}

\section{Instrumentos}

Diseñamos un cuestionario en el que se les preguntaba a niños y adolescentes sobre su participación en 26 actividades fuera de la escuela, y si estas suponían oportunidades de aprendizaje. La lista de actividades se obtuvo a través del análisis temático deductivo de 17 grupos focales que se llevaron a cabo previamente con participantes de 10 y 16 años y con sus padres y/o madres.

El objetivo principal del cuestionario fue explorar la participación en estas 26 actividades fuera de la escuela, examinar las características de los contextos sociales e institucionales en los que estas actividades tenían lugar, y qué aprendían en ellas. El cuestionario final se estructura en tres bloques de preguntas cerradas (ver Anexo). En el bloque inicial se solicita información a los estudiantes sobre algunas variables sociodemográficas, así como su edad y su género. En el segundo bloque se presenta la lista de 26 actividades y se pide a los estudiantes que indiquen si las realizan frecuentemente o no, y la importancia que tiene para ellos participar en las mismas. Igualmente, se les pide que describan las características de las actividades que realizan y consideran importantes, las razones por las que participan en ellas, y los dispositivos digitales que utilizan en estas actividades. Finalmente, en el tercer bloque se les pregunta cuánto piensan que aprenden en estas actividades fuera de la escuela, lo que piensan que aprenden en ellas y si estos aprendizajes son importantes para ellos. 
En este artículo presentamos, en primer lugar, los resultados de la primera pregunta del segundo bloque del cuestionario “¿Haces a menudo esta actividad?”, que incluye dos posibles respuestas: "sí" o "no". En la administración del cuestionario se explicitó a los participantes que "a menudo" significaba al menos una vez al mes. De este segundo bloque, también se presentan los resultados a la pregunta “¿Por qué motivos realizas esta actividad?”, cuyas opciones de respuesta, que podía ser múltiples, eran: "me divierte”, "me hace sentir bien”, "para aprender", "lo hacen las personas con las que estoy", "para complacer a mis padres", "me sentiría mal si no lo hiciera", "por obligación” y "en realidad no sé porque lo hago". Por último, se presentan los resultados de algunas preguntas de la tercera sección del instrumento: “¿Cuánto aprendes en esta actividad?” “ “¿Es importante lo que aprendes en esta actividad?”. Para ambas preguntas se planteaban tres opciones de respuesta: "nada", "algo" y "bastante".

\section{Procedimiento}

En primer lugar, como se ha indicado previamente, se realizaron grupos focales con estudiantes y familias que permitieron recoger las 26 actividades que los niños y adolescentes llevan a cabo habitualmente fuera de la escuela para tenerlas en cuenta en el diseño del cuestionario. Del mismo modo, previo a la aplicación del cuestionario, se llevó a cabo un estudio piloto en el cual el instrumento fue administrado a varios grupos de estudiantes de diferentes edades para evaluar su validez y fiabilidad. En este estudio piloto se comprobó la comprensión de las preguntas por parte de los participantes y en función de sus respuestas se llevaron a cabo algunos ajustes en la formulación de algunas frases. Se comprobó la validez aparente o "face valicity" (Cohen, Manion, y Morrison, 2000) preguntando a algunos participantes del estudio piloto sobre qué pensaban que evaluaba cada parte del cuestionario y si consideraban que lo evaluaba adecuadamente. Por lo que respecta a la validez interna, se calculó el alfa de Cronbach para cada uno de los elementos del cuestionario, dando como resultado .978 en el cuestionario dirigido a los participantes de 10 años y .966 para los de 16 años.

La versión final del cuestionario fue administrada durante el curso escolar 2014-15 a grupos clase completos de cada edad bajo la supervisión de sus tutores y de dos investigadores por aula. Recogimos los datos en la misma escuela a través de una aplicación online. Los estudiantes recibieron garantías de que sus respuestas serían anónimas y solo se utilizarían para los fines de la investigación, además, obtuvimos previamente, y gracias a la colaboración de 
los equipos directivos de los centros, el consentimiento informado por parte de los responsables legales de los participantes al ser estos menores de edad.

\section{Análisis de datos}

El análisis de los datos se realizó con el software estadístico SPSS v22. Utilizamos estadísticos descriptivos para calcular las frecuencias y los porcentajes de respuesta correspondientes al total de la muestra y respecto a las diferentes variables, y la prueba chicuadrado de Pearson para identificar si existían diferencias en la participación de los alumnos en actividades fuera de la escuela dentro de cada una de las variables sociodemográficas. El nivel de significación se estableció a p $\leq .05$, trabajándose con un nivel de confianza del 95\%.

\section{Resultados}

Participación en actividades fuera de la escuela y diferencias según las características sociodemográficas

Los resultados indican que entre las 26 actividades por las que se preguntaba hay una gran variedad respecto a la cantidad de participantes del estudio que dicen realizarlas. Por ello, hemos establecido y clasificado las actividades en cuatro rangos de participación basándonos en el porcentaje de estudiantes que informaron participar en cada una de las actividades (ver Tabla 2): actividades en las que más del $75 \%$ de los participantes indican que participan, actividades que realizan entre el 50 y el $74 \%$, actividades que realizan entre el 25 y el $49 \%$ y actividades en las que participan el $24 \%$ o menos de la muestra.

La lista de actividades está ordenada de forma decreciente en función del porcentaje de estudiantes que declaran participar en estas actividades (de mayor a menor participación). Esta clasificación de las 26 actividades en rangos se mantiene en las tablas 3 y 4 para facilitar la lectura e interpretación de las diferencias significativas encontradas. 
Tabla 2. Porcentaje de participación de los estudiantes en cada actividad

\begin{tabular}{|c|c|}
\hline Rangos de participación & Actividades y porcentaje de participantes \\
\hline $\begin{array}{l}\text { Actividades en las que } \\
\text { participan más del } 75 \% \text { de la } \\
\text { muestra }\end{array}$ & $\begin{array}{l}\text { Hacer deberes }(91 \%) \\
\text { Escuchar música }(87 \%) \\
\text { Hacer actividades físicas }(84 \%) \\
\text { Practicar un deporte }(83 \%) \\
\text { Ayudar en tareas del hogar }(81 \%) \\
\text { Mirar la televisión }(80 \%)\end{array}$ \\
\hline $\begin{array}{l}\text { Actividades en las que } \\
\text { participan entre el } 50 \% \text { y el } \\
74 \% \text { de la muestra }\end{array}$ & $\begin{array}{l}\text { Ir de compras }(72 \%) \\
\text { Participar en redes sociales }(72 \%) \\
\text { Hacer fotografías }(60 \%) \\
\text { Leer }(59 \%) \\
\text { Estudiar idiomas }(58 \%) \\
\text { Viajar }(58 \%) \\
\text { Cantar, tocar un instrumento, bailar }(53 \%) \\
\text { Cuidar animales }(52 \%) \\
\text { Jugar solo o con amigos }(50 \%)\end{array}$ \\
\hline $\begin{array}{l}\text { Actividades en las que } \\
\text { participan entre el } 25 \% \text { y el } \\
49 \% \text { de la muestra }\end{array}$ & $\begin{array}{l}\text { Ir al cine, teatro, otros espectáculos (49\%) } \\
\text { Pintar, dibujar, hacer manualidades (47\%) } \\
\text { Jugar a videojuegos }(46 \%) \\
\text { Cocinar }(41 \%) \\
\text { Hacer salidas a la naturaleza }(33 \%) \\
\text { Jugar a juegos de mesa }(33 \%) \\
\text { Estudiar música }(30 \%) \\
\text { Cuidar plantas }(28 \%)\end{array}$ \\
\hline $\begin{array}{l}\text { Actividades en las que } \\
\text { participan menos del } 24 \% \text { de } \\
\text { la muestra }\end{array}$ & $\begin{array}{l}\text { Coleccionar }(24 \%) \\
\text { Ir a museos, exposiciones, zoo }(24 \%) \\
\text { Ir a la iglesia, mezquita }(19 \%)\end{array}$ \\
\hline
\end{tabular}

La tabla 3 señala la existencia o no de diferencias significativas en los porcentajes de participación de los alumnos en las 26 actividades fuera de la escuela teniendo en cuenta cada una de las variables sociodemográficas estudiadas. Los resultados de la prueba chi-cuadrado señalan la presencia de diferencias en la participación en un mayor número de actividades en función del género y la edad de los estudiantes. 
Tabla 3. Diferencias significativas en la participación en función de la localización geográfica, el nivel socioeconómico, el género y la edad

\begin{tabular}{|c|c|c|c|c|c|}
\hline $\begin{array}{l}\text { Rangos de } \\
\text { participación }\end{array}$ & Actividad & $\begin{array}{c}\text { Localización } \\
\text { geográfica }\end{array}$ & $\begin{array}{c}\text { Nivel } \\
\text { socioeco- } \\
\text { nómico }\end{array}$ & Género & Edad \\
\hline \multirow{6}{*}{$\begin{array}{l}\text { Actividades en las } \\
\text { que participan más } \\
\text { del } 75 \% \text { de la } \\
\text { muestra }\end{array}$} & Hacer deberes & - & - & .000 & .000 \\
\hline & Escuchar música & - & - & .004 & .000 \\
\hline & Hacer actividades físicas & - & - & - & .049 \\
\hline & Practicar un deporte & - & - & .004 & - \\
\hline & Ayudar en tareas del hogar & - & - & .000 & .002 \\
\hline & Mirar la televisión & - & - & - & - \\
\hline \multirow{9}{*}{$\begin{array}{l}\text { Actividades en las } \\
\text { que participan } \\
\text { entre el } 50 \% \text { y el } \\
74 \% \text { de la muestra }\end{array}$} & Ir de compras & - & - & .000 & - \\
\hline & Participar en redes sociales & - & - & - & .000 \\
\hline & Hacer fotografías & - & - & .000 & - \\
\hline & Leer & - & - & .000 & .000 \\
\hline & Estudiar idiomas & - & - & .000 & - \\
\hline & Viajar & .019 & - & .030 & - \\
\hline & Cantar, tocar un instrumento, bailar & - & - & .000 & .011 \\
\hline & Cuidar animales & .000 & - & - & .000 \\
\hline & Jugar solo o con amigos & .043 & - & - & .000 \\
\hline \multirow{8}{*}{$\begin{array}{l}\text { Actividades en las } \\
\text { que participan } \\
\text { entre el } 25 \% \text { y el } \\
49 \% \text { de la muestra }\end{array}$} & Ir al cine, teatro, otros espectáculos & - & - & .000 & .003 \\
\hline & Pintar, dibujar, hacer manualidades & .047 & .004 & .000 & .000 \\
\hline & Jugar a videojuegos & .026 & - & .000 & .000 \\
\hline & Cocinar & - & - & .000 & - \\
\hline & Hacer salidas a la naturaleza & .039 & - & - & .000 \\
\hline & Jugar a juegos de mesa & - & - & - & .000 \\
\hline & Estudiar música & .003 & - & .042 & .000 \\
\hline & Cuidar plantas & - & - & - & .000 \\
\hline \multirow{3}{*}{$\begin{array}{l}\text { Actividades en las } \\
\text { que participan } \\
\text { menos del } 24 \% \text { de } \\
\text { la muestra }\end{array}$} & Coleccionar & - & - & .000 & .000 \\
\hline & Ir a museos, exposiciones, zoo & .008 & .009 & - & .000 \\
\hline & Ir a la iglesia, mezquita & - & .000 & - & .000 \\
\hline
\end{tabular}

Se observan diferencias significativas en función de la localización geográfica en seis actividades ("viajar", "cuidar plantas", "jugar solo o con amigos", "pintar, dibujar, hacer manualidades", "hacer salidas a la naturaleza" y "estudiar música”) en las que la proporción de estudiantes que participa es más elevada en áreas rurales, mientras que en otras dos actividades (“jugar a videojuegos” e "ir a museos, exposiciones, zoo”) la proporción más elevada corresponde a los estudiantes de áreas urbanas. Respecto al nivel socioeconómico, se observan diferencias menores: "pintar" e "ir a la iglesia o mezquita", estas son más frecuentes en estudiantes de niveles socioeconómicos bajos, mientras que "ir a museos, exhibiciones, zoo" 
es más frecuente en estudiantes de nivel socioeconómico alto. Destacamos también que en ambas variables las diferencias se han encontrado mayoritariamente en actividades en las que participa menos del $50 \%$ de la muestra.

Son más llamativas las diferencias observadas en función del género y la edad, que presentan significación en 16 y 19 actividades respectivamente. La tabla 4 presenta las frecuencias de participación desglosadas en función de las dos variables y sus correspondientes categorías. Se señala en negrita la dirección de las diferencias.

Tabla 4. Diferencias significativas en la participación en función del género y la edad

\begin{tabular}{|c|c|c|c|c|c|}
\hline $\begin{array}{l}\text { Rangos de } \\
\text { participación }\end{array}$ & Actividades & $\begin{array}{l}\% \\
\text { Femenino } \\
(\mathrm{n}=498)\end{array}$ & $\begin{array}{l}\% \\
\text { Masculino } \\
(\mathrm{n}=448)\end{array}$ & $\begin{array}{l}\% \\
10 \text { años } \\
(\mathrm{n}=411)\end{array}$ & $\begin{array}{l}\% \\
16 \text { años } \\
(\mathrm{n}=535)\end{array}$ \\
\hline \multirow{5}{*}{$\begin{array}{l}\text { Actividades en } \\
\text { las que } \\
\text { participan más } \\
\text { del } 75 \% \text { de la } \\
\text { muestra }\end{array}$} & Hacer deberes & 95,78 & 85,49 & 96,59 & 86,54 \\
\hline & Escuchar música & 90,16 & 83,93 & 75,43 & 96,26 \\
\hline & Hacer actividades físicas & - & - & 87,10 & 82,43 \\
\hline & Practicar un deporte & 80,12 & 87,05 & - & - \\
\hline & Ayudar en tareas del hogar & 87,55 & 73,21 & 76,16 & 84,30 \\
\hline \multirow{9}{*}{$\begin{array}{l}\text { Actividades en } \\
\text { las que } \\
\text { participan entre } \\
\text { el } 50 \% \text { y el } 74 \% \\
\text { de la muestra }\end{array}$} & Ir de compras & 82,93 & 60,71 & - & - \\
\hline & Participar en redes sociales & - & - & 44,77 & 92,15 \\
\hline & Hacer fotografías & 73,49 & 45,98 & - & - \\
\hline & Leer & 65,06 & 51,34 & 75,67 & 45,42 \\
\hline & Estudiar idiomas & 65,26 & 50,89 & - & - \\
\hline & Viajar & 61,45 & 54,46 & - & - \\
\hline & $\begin{array}{l}\text { Cantar, tocar un instrumento, } \\
\text { bailar }\end{array}$ & 66,27 & 38,17 & 57,66 & 49,35 \\
\hline & Cuidar animales & - & - & 61,31 & 44,67 \\
\hline & Jugar solo o con amigos & - & - & 70,32 & 34,58 \\
\hline \multirow{8}{*}{$\begin{array}{l}\text { Actividades en } \\
\text { las que } \\
\text { participan entre } \\
\text { el } 25 \% \text { y el } 49 \% \\
\text { de la muestra }\end{array}$} & $\begin{array}{l}\text { Ir al cine, teatro, otros } \\
\text { espectáculos }\end{array}$ & 54,82 & 41,52 & 43,07 & 52,71 \\
\hline & $\begin{array}{l}\text { Pintar, dibujar, hacer } \\
\text { manualidades }\end{array}$ & 53,61 & 39,06 & 72,02 & 27,29 \\
\hline & Jugar a videojuegos & 22,09 & $\mathbf{7 2 , 7 7}$ & 53,04 & 40,75 \\
\hline & Cocinar & 47,59 & 34,60 & - & - \\
\hline & Hacer salidas a la naturaleza & - & - & 55,72 & 27,48 \\
\hline & Jugar a juegos de mesa & - & - & 57,18 & 14,77 \\
\hline & Estudiar música & 33,33 & 27,23 & 42,34 & 21,31 \\
\hline & Cuidar plantas & - & - & 47,69 & 13,46 \\
\hline \multirow{3}{*}{$\begin{array}{l}\text { Actividades en } \\
\text { las que } \\
\text { participan } \\
\text { menos del } 24 \% \\
\text { de la muestra }\end{array}$} & Coleccionar & 18,88 & 29,24 & 44,28 & 8,04 \\
\hline & Ir a museos, exposiciones, zoo & - & - & 26,76 & 12,34 \\
\hline & Ir a la iglesia, mezquita & - & - & 27,49 & 11,96 \\
\hline
\end{tabular}


En relación con el género, los chicos tienden a participar más en "practicar un deporte", "jugar a videojuegos" y "coleccionar", mientras que las chicas prefieren las otras trece actividades. Sin embargo, es importante señalar que las mayores diferencias (más del $20 \%$ en la frecuencia de participación) se encuentran en actividades como "ir de compras", "hacer fotografías" y "cantar, tocar un instrumento, bailar", en las que se evidencia una mayor proporción de chicas implicadas, mientras que los chicos están sobrerrepresentados en "jugar a videojuegos" (72\% vs. $22 \%$ de chicas).

En relación con la edad, vemos cómo los estudiantes de 16 años participan especialmente en actividades como "escuchar música", "ayudar con las tareas del hogar", "participar en redes sociales" e "ir al cine, teatro, espectáculos", mientras que las otras que han mostrado significación son más frecuentes en los más jóvenes. Considerando las actividades con más del $20 \%$ de diferencia en frecuencia entre las dos edades, encontramos siete actividades que son más comunes en los estudiantes de 10 años, en contraste con dos actividades predominantes en los adolescentes: "escuchar música” y "participar en redes sociales" (esta última aumentando del $44 \%$ al $92 \%)$.

El valor subjetivo de los aprendizajes fuera de la escuela, y las diferencias según edad y género

Hemos analizado también el valor subjetivo que los estudiantes dan a su participación en actividades fuera de la escuela y de los aprendizajes que realizan en ellas. Para ello, hemos explorado: a) hasta qué punto el alumnado considera que ha aprendido como consecuencia de su participación en las diferentes actividades de fuera de la escuela (ver Tabla 5), b) qué importancia le dan a lo que aprenden en ellas (ver Tabla 6), y c) los motivos por los que participan en dichas actividades. Recordemos que en este bloque del instrumento los participantes respondieron las preguntas únicamente por las actividades en las que habían declarado anteriormente que participaban a menudo y que consideraban importantes; el resto de las actividades fueron excluidas. Los resultados que presentamos a continuación, en las tablas 5 y 6 , incluyen las actividades que en el conjunto de los datos corresponden a más del $75 \%$ de la frecuencia de respuestas de los participantes, razón por la que en las comparativas aparecen solo seis actividades. 
Tabla 5. Lista de las seis actividades en las que los niños y adolescentes consideran que han aprendido más, y diferencias según la edad y el género

\begin{tabular}{|c|c|c|c|c|}
\hline & $\begin{array}{l}\text { Masculino } \\
\text { de } 10 \text { años }\end{array}$ & $\begin{array}{l}\text { Femenino } \\
\text { de } 10 \text { años }\end{array}$ & $\begin{array}{l}\text { Masculino } \\
\text { de } 16 \text { años }\end{array}$ & $\begin{array}{l}\text { Femenino } \\
\text { de } 16 \text { años }\end{array}$ \\
\hline 1 & $\begin{array}{l}\text { Hacer deberes } \\
(90,6 \%)\end{array}$ & $\begin{array}{l}\text { Estudiar idiomas } * * \\
(96,2 \%)\end{array}$ & $\begin{array}{l}\text { Estudiar idiomas } \\
(100 \%)\end{array}$ & $\begin{array}{l}\text { Estudiar idiomas } \\
(100 \%)\end{array}$ \\
\hline 2 & $\begin{array}{l}\text { Practicar un deporte } \\
(86 \%)\end{array}$ & Hacer deberes $(95,6 \%)$ & Leer $(99,1 \%)$ & Viajar* $(99,5 \%)$ \\
\hline 3 & $\begin{array}{l}\text { Estudiar idiomas } \\
(83,4 \%)\end{array}$ & $\begin{array}{l}\text { Practicar un deporte } \\
(85,7 \%)\end{array}$ & $\begin{array}{l}\text { Hacer deberes } \\
(98,9 \%)\end{array}$ & $\begin{array}{l}\text { Hacer deberes } \\
(99,2 \%)\end{array}$ \\
\hline 4 & Viajar $(76,6 \%)$ & $\begin{array}{l}\text { Cuidar animales } \\
(84,1 \%)\end{array}$ & Viajar $(96,2 \%)$ & Leer $(97,9 \%)$ \\
\hline 5 & $\begin{array}{l}\text { Hacer salidas a la } \\
\text { naturaleza }(76,2 \%)\end{array}$ & Leer** $(83,1 \%)$ & $\begin{array}{l}\text { Practicar un deporte } \\
(95,8 \%)\end{array}$ & $\begin{array}{l}\text { Escuchar música*** } \\
(97 \%)\end{array}$ \\
\hline 6 & $\begin{array}{l}\text { Cuidar animales } \\
(73,4 \%)\end{array}$ & Viajar $(77,4 \%)$ & $\begin{array}{l}\text { Escuchar música } \\
(92 \%)\end{array}$ & $\begin{array}{l}\text { Practicar un deporte } \\
(96 \%)\end{array}$ \\
\hline
\end{tabular}

${ }^{*} p<.05 ; * * p<.01 ; * * * *<.001$

En términos generales, la Tabla 5 indica que los niños y adolescentes perciben que aprenden más en aquellas actividades fuera de la escuela generalmente vinculadas a lo que se hace en el contexto escolar (“estudiar idiomas", "hacer deberes", "leer"), si bien también mencionan otro tipo de actividades como "practicar un deporte", "viajar" y "escuchar música". Las actividades fuera de la escuela más valoradas en función de la cantidad de aprendizaje experimentado son casi las mismas en ambas edades y géneros, a excepción del caso de "practicar un deporte" y "cuidar animales" a los 10 años, y “escuchar música” a los 16 años. Sin embargo, aparecen algunas diferencias significativas en la cantidad de aprendizaje percibido en función de la edad y el género. Así, para el grupo de 10 años las diferencias se aprecian en dos actividades en las que las chicas consideran que han aprendido más cosas que los chicos ( $p=.019$ para "leer", y $p=.017$ para "estudiar idiomas"). A los 16 años las chicas también informan que aprenden más que los chicos en "viajar" ( $p=.021)$ y "escuchar música" $(p=.004)$. Es importante señalar que a esta edad las tasas de elección de las respuestas superan el $90 \%$ en las seis actividades.

La importancia otorgada a los aprendizajes realizados en las actividades fuera de la escuela también presenta diferencias significativas de acuerdo con la edad y el género (ver 
Tabla 6). Las razones para escoger una actividad en particular también se mencionan, a pesar de que los alumnos podían señalar múltiples respuestas.

Tabla 6. Lista de las seis actividades fuera de la escuela en las que los niños y adolescentes consideran que lo que han aprendido es muy importante de acuerdo con la edad y el género

\begin{tabular}{|c|c|c|c|c|}
\hline & $\begin{array}{l}\text { Masculino } \\
\text { de } 10 \text { años }\end{array}$ & $\begin{array}{l}\text { Femenino } \\
\text { de } 10 \text { años }\end{array}$ & $\begin{array}{l}\text { Masculino } \\
\text { de } 16 \text { años }\end{array}$ & $\begin{array}{l}\text { Femenino } \\
\text { de } 16 \text { años }\end{array}$ \\
\hline 1 & $\begin{array}{l}\text { Estudiar idiomas } \\
(97,9 \%)\end{array}$ & $\begin{array}{l}\text { Estudiar idiomas } \\
(98,1 \%)\end{array}$ & $\begin{array}{l}\text { Estudiar idiomas } \\
(96,3 \%)\end{array}$ & $\begin{array}{l}\text { Estudiar idiomas* } \\
(98,7 \%)\end{array}$ \\
\hline 2 & $\begin{array}{l}\text { Hacer deberes } \\
(97,7 \%)\end{array}$ & Hacer deberes $(97 \%)$ & $\begin{array}{l}\text { Hacer deberes } \\
(91,6 \%)\end{array}$ & $\begin{array}{l}\text { Hacer deberes } \\
(93,4 \%)\end{array}$ \\
\hline 3 & $\begin{array}{l}\text { Cuidar plantas } \\
(96,2 \%)\end{array}$ & Viajar $(95,4 \%)$ & Leer $(83,5 \%)$ & Viajar** $(88,4 \%)$ \\
\hline 4 & Viajar (96\%) & Leer $^{*}(93,1 \%)$ & Viajar $(80,4 \%)$ & Cocinar $(80,9 \%)$ \\
\hline 5 & $\begin{array}{l}\text { Practicar un deporte } \\
* * *(95,2 \%)\end{array}$ & $\begin{array}{l}\text { Cuidar animales } \\
(91,7 \%)\end{array}$ & $\begin{array}{l}\text { Practicar un deporte } \\
(78,9 \%)\end{array}$ & Leer $(79,9 \%)$ \\
\hline 6 & $\begin{array}{l}\text { Hacer salidas a la } \\
\text { naturaleza }(93,9 \%)\end{array}$ & Cuidar plantas $(91 \%)$ & $\begin{array}{l}\text { Ayudar en tareas del } \\
\text { hogar** }(75,7 \%)\end{array}$ & $\begin{array}{l}\text { Practicar un deporte } \\
(76,7 \%)\end{array}$ \\
\hline
\end{tabular}

$* p<.05 ; * * p<.01 ; * * * p<.001$

$\mathrm{Al}$ igual que en la tabla 5, los resultados de la tabla 6 muestran que las actividades fuera de la escuela que están más vinculadas a lo que se hace en el contexto escolar son las más valoradas por los estudiantes en términos de la importancia del aprendizaje obtenido. La razón principal para elegir estas actividades a los 10 años es "para aprender" (el 73\% de los participantes y sin diferencias significativas entre chicos y chicas). Sin embargo, a esta edad también señalan que "hacer deberes" es algo que hacen "por obligación" (el 34\% de las chicas frente el $34,5 \%$ de los chicos, sin diferencias significativas -n.s.) o para "complacer a mis padres" (el 27\% de las chicas frente el 14,4\% de los chicos, $p=.002$ ). Los motivos para realizar la actividad "estudiar idiomas" suelen ser "me hace sentir bien" (el 46\% de chicas frente al $31 \%$ de los chicos, n.s.) o, en especial las chicas, "para complacer a mis padres" (el 21,7\% de las chicas frente solo un $8,3 \%$ de los chicos, $p=.008)$.

A los 16 años, ambos géneros responden que "hacen deberes" fundamentalmente "por obligación" (el 76,6\% de las chicas frente el 72,4\% de los chicos, n.s.) o bien "para aprender" 
(el 75\% de las chicas frente el 73\% de los chicos, n.s.). En la actividad "estudiar idiomas" hay diferencias significativas, siendo las chicas las que más la valoran. Cuando reportan los motivos por los que realizan esta actividad, la mayoría responden hacerla "para aprender" (el 92\% de las chicas frente al 83\% de los chicos, $p=.007$ ), seguida de lejos de "me hace sentir bien" (el $25,8 \%$ de las chicas frente al 15,4\% de los chicos, $p=.014$ ) y "me divierte" (el 24,5\% de las chicas frente al $13 \%$ de chicos, $p=.005)$. Las otras cuatro actividades de la lista muestran un patrón de selección acorde con la edad de los estudiantes y dentro del mismo género.

Aparecen pues algunas diferencias significativas en las impresiones que tienen los participantes acerca del valor del aprendizaje en algunas actividades particulares fuera de la escuela. A los 10 años los chicos valoran más que las chicas "practicar un deporte" (un 95\% frente al 89\%, $p=.041)$, mientras que las chicas consideran que "leer" es la actividad fuera de la escuela más importante en términos de lo que aprenden en ella (93\% frente al 86\%, $p=.038$ ). Cuando se analizan las razones para escoger estas actividades, ambos géneros aluden a razones intrínsecas. Específicamente para "leer" los motivos que exponen son "me divierte" (70\% tanto en chicos como en chicas, n.s.) y "para aprender" (el 61,5\% de chicas frente al 58,5\% de los chicos, $p=.028$ ). Y en el caso de "practicar un deporte", las razones son las mismas para ambos sexos y es porque "me divierte" ( $88,8 \%$ de chicas frente al $87 \%$ de chicos, n.s.).

También en el grupo de adolescentes de 16 años aparecen diferencias significativas en la importancia que otorgan a las actividades fuera de la escuela en función del género. En concreto en las actividades "estudiar idiomas", "viajar" y "ayudar en tareas del hogar", las chicas dan mayor importancia que los chicos a lo que aprenden en "estudiar idiomas" $(p=.001)$ y "viajar" ( $p=.005)$, mientras que los chicos valoran más lo que aprenden mientras "ayudan en tareas del hogar" ( $p=.001)$. Si se examinan las razones para escoger estas actividades, vemos que para "estudiar idiomas" ambos géneros aluden a razones intrínsecas, aunque las chicas de forma más contundente: primero para "aprender" (el 91,7\% de chicas frente al 82,7\% de los chicos, $p=.007$ ), seguido de "me hace sentir bien" (el 25,8\% de chicas frente al 15,4\% de los chicos, $p=.014$ ) y de "me divierte" (el 24,5\% de chicas frente al 13\% de los chicos, $p=$ .005). Las chicas también valoran de forma más positiva "viajar" como una actividad que "me divierte" (el 94\% de chicas frente al 89\% de los chicos, $p=.042$ ), seguida de "me hace sentir bien" (el 77\% de chicas frente al 62\% de los chicos, $p=.002)$ y "para aprender" (57\% de chicas frente al 54\% de chicos), aunque en este último motivo no hay diferencias significativas. En contraste, algunas actividades se hacen debido a motivos externos, como por ejemplo "ayudar 
en tareas del hogar". En este caso un número más elevado de chicas reporta hacerlas "por obligación" (el 66\% frente al 55\%, $p=.018$ ) y ambos géneros para "complacer a mis padres" (61\% frente al $54 \%$, n.s.).

En resumen, el análisis de la valoración subjetiva del aprendizaje asociado a actividades fuera de la escuela señala una relación directa entre la cantidad de aprendizaje percibido y la importancia otorgada a estos aprendizajes. En general, la mayoría de los participantes, pero sobretodo las chicas, tienden a dar importancia a la participación en actividades más vinculadas con lo que se hace en el contexto escolar. En contraste, los chicos suelen dar mayor importancia a las actividades relacionadas con el deporte, aunque las diferencias en las opiniones de chicos y chicas tienden a desaparecer a los 16 años.

\section{Discusión y conclusiones}

En el estudio que se ha presentado en este artículo se han explorado las diferencias en la participación de los niños y adolescentes en actividades fuera de la escuela en función de cuatro variables sociodemográficas. Si bien las investigaciones previas han puesto el foco en el estudio de actividades extraescolares dirigidas y con cierto grado de organización (por ejemplo, Barber et al., 2009; Bruyn y Cillessen, 2008; Feldman y Matjasko, 2005; Kadar-Satat, 2014; Simpkins et al., 2005), la selección de las 26 actividades de nuestro estudio incluye una diversidad de actividades que varían en su grado de formalidad y organización, y que pueden darse en diferentes contextos fuera de la escuela. Por otro lado, en su elección se ha considerado no solo la participación, sino el punto de vista de los estudiantes respecto al valor que le otorgan al aprendizaje, su importancia y los motivos para su participación.

En relación con el primer objetivo, los análisis realizados muestran diferencias destacadas en función de la edad y el género de los participantes, mientras que variables como el nivel socioeconómico y el área de residencia tienen una influencia mucho más limitada en menos de un tercio de las actividades fuera de la escuela estudiadas, y en la mayor parte de esas actividades participa menos del $50 \%$ de la muestra.

Según el área de residencia, como era esperado, los resultados indican que las características del contexto en el que los estudiantes viven determinan, en parte, su participación en ciertas actividades. Por ejemplo, los estudiantes que viven en zonas rurales parecen sacar 
más provecho de su entorno natural al aire libre (por ejemplo, "hacer salidas a la naturaleza", "cuidar animales", “jugar solo o con amigos"), mientras que los que viven en zonas urbanas tienen mayores oportunidades para participar en actividades culturales ("ir a museos, exhibiciones, zoo"). También aparecen diferencias en la participación de acuerdo con el nivel socioeconómico, aunque mucho menos marcadas y muy puntuales. Así, a diferencia de otros estudios que han señalado la inequidad en el acceso a distintas actividades extraescolares en función del nivel socioeconómico (por ejemplo, Kadar-Satat, 2014; Lareau, 2011; Simpkins et al., 2005), en nuestra muestra esta variable solo presenta diferencias en relación con tres actividades ("pintura, dibujo, manualidades"; "ir a la iglesia/mezquita"; "ir a museos, exhibiciones, zoo”), las dos primeras más frecuentes en alumnos con nivel socioeconómico bajo, y la última más frecuente en alumnos de nivel socioeconómico alto. Creemos que estas diferencias con otros estudios pueden deberse a dos factores: a) las características de las 26 actividades analizadas, ya que en el foco de los otros estudios revisados han considerado las actividades extraescolares dirigidas, mientras que en nuestro caso las actividades contempladas tienen grados variables de formalidad e institucionalización, y la mayoría no supone ningún coste económico para las familias; y b) el tipo de análisis realizado, ya que el uso de categorías generales (nivel socioeconómico alto o bajo) tiende a homogeneizar ciertas características de la muestra.

En contraste, las variables edad y género son las que tienen más incidencia en nuestros datos. En primer lugar, parece que la participación de los alumnos en actividades fuera de la escuela presenta un patrón de desarrollo de acuerdo con la edad. Así, actividades como "escuchar música” y "participar en redes sociales" son mucho más frecuentes a los 16 años, mientras que los niños de 10 años participan en un rango de actividades más amplio. También observamos cómo algunas actividades relacionadas con lo que se hace en el contexto escolar disminuyen en la adolescencia (por ejemplo, "leer" pasa del 75\% a los 10 años a solo el 45\% a los 16), mientras que otras se mantienen más estables (por ejemplo, "hacer deberes" baja del $96 \%$ al $86 \%$, si bien es la actividad fuera de la escuela más frecuente que reportan los estudiantes). La tendencia opuesta se observa en actividades como "participar en redes sociales", que tiene un marcado incremento a medida que los estudiantes se hacen mayores. Nuestros resultados están en línea con los de Engel et al. (2018), quienes encontraron además que la mayoría de los alumnos usaban las nuevas tecnologías fuera de la escuela para el entretenimiento, o para comunicarse entre ellos, más que para aprender. 
En segundo lugar, nuestro estudio señala diferencias marcadas en la participación en actividades fuera de la escuela en función del género. Al igual que en estudios previos en España (Molinuevo, 2009; Nuviala et al., 2009) y en los Estados Unidos (Barber et al., 2009; Bruyn y Cillessen, 2008; Feldman y Matjasko, 2005; Simpkins et al., 2005), la participación de los chicos y chicas de la muestra señala ciertas tendencias estereotipadas en función del género. Es preocupante que en ambas edades los chicos estén sobrerrepresentados en actividades como "practicar un deporte" y "jugar a videojuegos", mientras que las chicas demuestran tener una mayor preferencia para participar en actividades de tipo social o relativas a lo escolar. Nuestros resultados son parecidos a los de Bruyn y Cillessen (2008), quienes encontraron una sobrerrepresentación de los chicos en actividades extraescolares dedicadas al deporte y a los coches, y al uso del ordenador, mientras que las chicas estaban sobrerrepresentadas en actividades extraescolares donde se trabajaban aspectos sociales y creativos. Vale la pena señalar que aparecen las mismas tendencias a pesar de que nuestro estudio solo ha explorado 26 actividades muy diversas fuera la escuela, en contraste con las 70 actividades extraescolares que sondearon Bruyn y Cillessen (2008), y que nuestros datos son mucho más recientes, lo cual sugiere la fuerza de los discursos dominantes acerca del género y su prevalencia a lo largo del tiempo.

De acuerdo con la perspectiva de la práctica social (Dreier, 1997, citado en DiGiacomo et al., 2018), "la implicación personal y la participación en un contexto depende de su estatus en relación con su trayectoria de participación en otros contextos” (p. 53). Por lo tanto, el deseo de una persona por participar en una determinada actividad fuera de la escuela puede tener efectos facilitadores, o bien limitadores, en la forma y el resultado que tengan las siguientes experiencias de aprendizaje. En nuestro estudio en particular, parece que ciertos estereotipos relacionados con el género tienen un fuerte impacto en las decisiones de los chicos y chicas a la hora de decidir participar en determinadas actividades fuera de la escuela y, por lo tanto, pueden ejercer una influencia en sus experiencias de aprendizaje dentro de la escuela y su futura trayectoria académica y profesional (Eccles, 2014).

Como señalan Philip y Azevedo (2017), es crucial tener en cuenta las dimensiones socioeconómicas y sociopolíticas del aprendizaje. Así, creemos que la presencia de diferencias significativas en la participación de chicos y chicas en las 26 actividades de nuestro estudio indican la presencia de ciertas limitaciones a nivel contextual que pueden conducir a desigualdades de acceso y participación en ciertas actividades formativas en detrimento de 
otras. Desde nuestra perspectiva, estas desigualdades de acceso a ciertas actividades fuera de la escuela tienen efectos importantes en las experiencias de aprendizaje de los jóvenes, y también en el desarrollo de habilidades para su aprendizaje a lo largo de la vida. Dado que estar bien preparado para el futuro depende del desarrollo de estas habilidades en distintos contextos, cuando el acceso al aprendizaje se limita a la participación en ciertas actividades fuera de la escuela, también lo hace el desarrollo de competencias y habilidades para aprender "a lo largo" y "a lo ancho" de la vida (en distintos contextos de uso).

También es preocupante que entre los participantes del estudio haya un consenso total en lo que cuenta como "aprendizaje". En ambas edades, hay una relación clara entre la cantidad de aprendizaje percibido y el valor subjetivo que tienen los aprendizajes realizados en actividades fuera de la escuela relacionadas con contenidos escolares. Sin embargo, esta relación es menos clara cuando se habla del resto de actividades de tipo informal. Los resultados de las tablas 5 y 6 indican que los estudiantes realizan una clara distinción entre las actividades fuera de la escuela que proporcionan "aprendizajes valiosos" (aquellos que se relacionan con contenidos o habilidades relacionadas o más cercanas a las del contexto escolar), en contraste con otras actividades que proporcionan lo que hemos denominado "habilidades para la vida" (como viajar, practicar un deporte o ayudar en tareas del hogar). Este dato sugiere que las experiencias escolares tienen una gran influencia en el valor que se otorga a ciertos tipos de actividades fuera de la escuela en detrimento de otras.

Los motivos por los que los estudiantes participan en estas actividades que proporcionan "aprendizajes valiosos" varían desde razones intrínsecas ("para aprender", "me hace sentir bien", o "me divierte") a razones extrínsecas ("por obligación", o "para complacer a mis padres"). Parece que los discursos sobre el aprendizaje escolar están apoyados y reforzados por los padres, ya que son elementos clave para promover la percepción de los estudiantes sobre qué tipo de actividades fuera de la escuela son más "provechosas" en términos de aprendizaje. Siguiendo una perspectiva sociocultural, el aprendizaje se evidencia a través de la participación cambiante de las personas en las actividades significativas de sus comunidades, y se apoya con y a través de las relaciones sociales y materiales que alientan a los estudiantes a participar en nuevos roles y responsabilidades a medida que se van apropiando de las prácticas que se valoran en su contexto sociocultural. 
De acuerdo con nuestros resultados, creemos necesario que las escuelas y los institutos no solo proporcionen oportunidades y recursos para el aprendizaje, sino que también promuevan la reflexión de los estudiantes sobre la importancia de participar en una amplia variedad de contextos no formales e informales fuera de escuela para conseguir alcanzar sus objetivos personales de aprendizaje, tanto presentes como futuros (Leander, Phillips y Taylor, 2010; Coll, 2013, 2018; Sefton-Green, 2012). Al igual que otros autores, también creemos que las instituciones educativas son los contextos más adecuados para proporcionar este tipo de mensajes. Sin embargo, deben ser conscientes del riesgo que tanto los estereotipos de género (Mosteiro y Porto, 2017; Colás y Villaciervos, 2007) como los discursos sociales sobre el aprendizaje formal pueden tener en la toma de decisiones de los estudiantes. Se deben implementar nuevas formas de validar las experiencias de aprendizaje no formales e informales para reconocer y aumentar la visibilidad social de otras experiencias formativas desarrolladas fuera de los contextos formales (Cedefop, 2016, p.6).

También queremos poner en valor la importancia de aprender en distintos contextos, ya que los niños conectarán de forma deliberada distintas experiencias de aprendizaje adquiridas en una amplia gama de contextos con diferentes actores, entornos y motivos a lo largo de sus vidas (Bronkhorst y Akkerman, 2016; Erstad et al., 2016). A este respecto, creemos que las experiencias subjetivas de aprendizaje y en particular los motivos que sustentan la participación de los estudiantes en ciertas actividades fuera de la escuela cobran gran importancia. Comprender cómo y porqué los niños y adolescentes participan en distintas actividades de aprendizaje fuera de la escuela debería ayudar a las instituciones de educación formal a conectar sus experiencias de aprendizaje de una manera positiva y ayudar a los jóvenes a atribuir sentido al aprendizaje que adquieren en distintos contextos. El objetivo principal debería ser el poder ayudar a los estudiantes a reconstruir su visión del aprendizaje para que vaya más allá del contexto formal, y garantizar la equidad (Bronkhorst y Akkerman, 2016; Coll, 2013, 2018; Leander, Philips y Taylor, 2010).

Finalmente, señalar como limitaciones del estudio que el abordaje metodológico utilizado solo nos ha permitido ofrecer un retrato general de la participación de los niños y jóvenes en actividades fuera de la escuela y sus motivos. Vemos necesario, pues, el desarrollo de futuras investigaciones de corte etnográfico que permitan conocer en mayor profundidad las razones de la participación de los jóvenes en determinadas actividades fuera de la escuela, y que presten atención a las dimensiones sociales, económicas y políticas implicadas en esta 
participación. Estos estudios deberían contemplar el análisis de actividades que puedan realizarse en distintos contextos de aprendizaje a la vez (y, por lo tanto, que incorporen actividades mediadas por el uso de las TIC), y que contemplen el peso que tienen aspectos como la cultura de origen, el tiempo de residencia en un país, o la pertenencia a una determinada comunidad de práctica o de intereses. También son necesarios más estudios longitudinales que utilicen entrevistas en profundidad para explorar las experiencias subjetivas de aprendizaje de los niños y adolescentes en diferentes contextos, tanto dentro como fuera de la escuela, y sus conexiones. Las futuras investigaciones sobre estos temas ayudarán a los agentes educativos (tanto maestros, educadores, como miembros de la familia y la comunidad), a la administración y a los políticos educativos a ser conscientes de la importancia del desarrollo de habilidades para funcionar a lo largo y ancho de la vida, y a introducir cambios a distintos niveles para luchar contra las desigualdades sociales, culturales y educativas de las personas.

\section{Agradecimientos}

Este trabajo ha sido apoyado por el Ministerio de Economía y Competitividad en el marco de los proyectos EDU2013-40965-R y EDU2017-82321-R.

\section{Referencias}

Banks, J.A., Au, K.H., Ball, A.F., Bell, P., Gordon, E.W., Gutiérrez, K.D., ... Zhou, M. (2007). Learning in and out of School in Diverse Environments: Life-long, Life-wide, Life-deep. Seattle: The LIFE Center, University of Washington.

Barber, B.L., Abbott, B.D., Blomfield, C.J., \& Eccles, J.S. (2009). Secrets of their success: Activity Participation and Positive Youth Development. In M.J., Furlong, R. Gilman, \& E.S. Huebner (eds.), Handbook of Positive Psychology in Schools (pp.273-289). New York: Routledge.

Barron, B. (2006). Interest and Self-sustained Learning as a Catalyst of Development: A Learning Ecologies Perspective. Human Development, 49, 193-224. DOI:10.1159/000094368

Bouffard, S., Little, P., \& Weiss, H. (2006). Building and evaluating out-of-school time connections. The Evaluation Exchange, 12(1-2), 2-6.

Bronkhorst, L.H., \& Akkerman, S.F. (2016). At the Boundary of School: Continuity and Discontinuity in Learning Across Contexts. Educational Research Review, 19, 18-35. DOI:10.1016/j.edurev.2016.04.001

Bruyn, E. H., \& Cillessen, A.H. (2008). Leisure activity preferences and perceived popularity in early adolescence. Journal of Leisure Research, 40(3), 442-457. 
Participación en actividades fuera de la escuela y su valor subjetivo: un estudio exploratorio con niños y adolescentes

Cedefop (European Centre for the Development of Vocational Training). (2016). European guidelines for validating non-formal and informal learning. Luxembourg: Publications Office. Cedefop reference series, No 104. DOI: 10.2801/008370

Cohen, L., Manion, L., \& Morrison, K. (2000). Research methods in education. London: Routledge and Falmer.

Colás, P. y Villaciervos, P. (2007). La interiorización de los estereotipos de género en jóvenes y adolescentes. Revista de Investigación Educativa, 1(25), 35-58. Retrieved from: $\underline{\text { https://revistas.um.es/rie/article/view/96421 }}$

Coll, C. (2009). Enseñar y aprender en el siglo XXI: el sentido de los aprendizajes escolares. En A. Marchesi, J.C. Tedesco, y C. Coll (Eds.), Reformas educativas y calidad de la educación (pp.101-112). Madrid: OEI Santillana.

Coll, C. (2013). La educación formal en la nueva ecología del aprendizaje: tendencias, retos y agenda de investigación. En J.L. Rodríguez Illera (Comp.), Aprendizaje y Educación en la Sociedad Digital (pp. 156-170). Barcelona: Universitat de Barcelona. DOI: 10.1344/106.000002060

Coll, C. (2016). La personalització de l'aprenentatge escolar. El què, el per què i el com d'un repte indefugible. En J. M. Vilalta (Dr.). Reptes de l'educació a Catalunya. Anuari d’Educació 2015 (pp.45104). Barcelona: Fundació Bofill. [Traducción al castellano disponible en: http://psyed.edu.es/archivos/grintie/Coll_2016_LaPersonalizaci\%C3\%B3nDelAprendizajeEscolar.pdf

Coll, C. (2018). La personalización del aprendizaje escolar, una exigencia de la nueva ecología del aprendizaje. En C. Coll (Coord.), Personalización del aprendizaje. (pp. 5-11). Barcelona: Editorial Graó.

Darling, N. (2005). Participation in extracurricular activities and adolescent adjustment: Cross-sectional and longitudinal findings. Journal of Youth and Adolescence, 34, 493-505. DOI:10.1007/s10964-005-72668

Davaki, K. (2016). Differences in Men's and Women's Work, Care and Leisure Time. Directorate General for Internal Policies. European Parliament's Comittee on Women's Rights and Gender Equality. Policy Department C: Citizens' Rights and Constitutional Affairs. Retrieved from: http://www.europarl.europa.eu/committees/en/supporting-analyses-search.html

DiGiacomo, D. K., Van Horne, K., Van Steenis, E., \& Penuel, W. R. (2018). The material and social constitution of interest. Learning, culture and social interaction, 19, 51-60. DOI: 10.1016/j.1csi.2018.04.010

Eccles, J.S. (2014). Gendered Socialization of STEM Interests in the Family. International Journal of Gender, Science and Technology, 7(2), 116-132. Retrieved from: http://genderandset.open.ac.uk/index.php/genderandset/article/view/419/692

Engel, A., Coll, C., Membrive, A., y Oller, J. (2018). Information and communication technologies and students' out-of-school learning experiences. Digital Education Review, 33, 130-149. Recuperado de: http://revistes.ub.edu/index.php/der/article/view/21689

Engel, A., Fauré, J., Membrive, A., Merino, I., y Coll, C. (2019). The influence of parents in the discursive construction of technology-mediated learning experiences. Mind, Culture and Activity, 26(4), 323-335. DOI: $10.1080 / 10749039.2019 .1685549$ 
Judith Oller et al.

Erstad, O., Kumpulainen, K., Mäkitalo, Å, Schrøder, K.C., Pruulmann-Vengerfeldt, P., \& Thuridur, J. (2016). Learning Across Contexts in the Knowledge Society. Amsterdam: Sense Publishers.

Feldman, A.F., \& Matjasko, J.L. (2005). The Role of School-Based Extracurricular Activities in Adolescent Development: A Comprehensive Review and Future Directions. Review of Educational Research, 75(2), 159-210. DOI:10.3102/00346543075002159

Holland, D., \& Lave, J. (2009). Social Practice Theory and the Historical Production of Persons. Actio: An International Journal of Human Activity Theory, 2, 1-15

Kadar-Satat, G. (2014). Participation in out-of-school activities and the socio-economic gap in children's academic outcomes (Unpublished thesis dissertation). The University of Edinburgh, Scotland.

Lareau, A. (2011). Unequal Childhoods: Class, Race and Family Life (2nd.ed.). Berkeley, C.A: University of California Press.

Leander, K.M., Phillips, N.C., \& Taylor, K.H. (2010). The Changing Social Spaces of Learning: Mapping New Mobilities. Review of Research in Education, 34(1), 329-394. DOI:10.3102/0091732X09358129

Mahoney, J.L., Vandell, D.L., Simpkins, S., \& Zarrett, Z. (2009). Adolescents' out-of-school activities. In L. Lerner, \& L. Steinberg (Eds.), Handbook of adolescent psychology (3rd. ed., Vol 2, pp. 228-269). New York: Wiley.

Molinuevo, B. (2009). El temps fora de l'horari lectiu: els recursos a la llar, les activitats i els agents educatius. En R. Torrubia, \& E. Doval, (Eds.), Família i Educació a Catalunya (pp. 122-195). Barcelona: Fundació Jaume Bofill. Retrieved from: https://www.fbofill.cat/publicacions/familia-i-educacio-catalunya

Mosquera, M.J. y Puig, N. (2002). Género y edad en el deporte. In M. García Ferrando, N. Puig, \& F. Lagardera (Eds.), Sociología del Deporte (pp. 114-141). Madrid: Alianza.

Mosteiro, M.J. y Porto, A.M. (2017). Análisis de los estereotipos de género en alumnado de formación profesional: diferencias según sexo, edad y grado. Revista de Investigación Educativa, 35(1), 151-165. DOI:10.6018/rie.35.1.257191

Nuviala, A., Murguía, D., Fernández, A., Ruiz, F., \& García, M.E. (2009). Typologies of Occupation of Leisuretime of Spanish Adolescents: The Case of the Participants in Physical Activities Organized. Journal of Human Sport and science, 4(1), 29-39. DOI:10.4100/jhse.2009.41.04

Philip, T.M., \& Azevedo, F.S. (2017). Everyday science learning and equity: Mapped and contested terrain. Science Education, 101 (4), 526-532.

Sefton-Green, J. (2012). Learning at Not-School. A review of study, theory and advocacy for education in nonformal settings. Cambridge: MIT Press.

Shulruf, B. (2010). Do extra-curricular activities in schools improve educational outcomes? A critical review and meta-analysis of the literature. International Review of Education, 56(5-6), 591-612. DOI:10.1007/s11159-010-9180-X 
Participación en actividades fuera de la escuela y su valor subjetivo: un estudio exploratorio con niños y adolescentes

Simpkins, S.D., Ripke, M., Huston, A.C., \& Eccles, J.S. (2005). Predicting participation and outcomes in out-ofschool activities: similarities and differences across social ecologies. New Directions for Youth Development, 105, 51-70. DOI:10.1002/yd.107

Trilla, J., Gros, B., López F. y Martín, M. J. (2003). La educación fuera de la escuela. Ámbitos no formales y Educación Social. Barcelona: Ariel. 


\section{Anexo}

Estructura y contenido del cuestionario

\begin{tabular}{ll}
\hline $\begin{array}{l}\text { Primer bloque: } \\
\text { Información sociodemográfica }\end{array}$ & $\begin{array}{l}\text { Segundo bloque: } \\
\text { Actividades fuera de la escuela }\end{array}$ \\
& \\
\hline P1.1. Escuela & P2.1. ¿Haces a menudo esta actividad? \\
P1.2. Fecha de nacimiento & P2.2. ¿Cómo de importante es para ti \\
P1.3. Sexo & esta actividad? \\
P1.4. País de nacimiento & P2.3. ¿Dónde realizas esta actividad? \\
P1.5. Si no has nacido en España, & P2.4. ¿Con quién haces esta actividad? \\
¿cuántos años llevas viviendo aquí? & P2.5. ¿Por qué motivos realizas esta \\
P1.6. Lengua familiar & actividad? \\
P1.7. País de origen de la madre & P2.6. ¿Qué dispositivos electrónicos \\
P1.8. País de origen del padre & utilizas cuando participas en esta \\
& actividad?
\end{tabular}

\section{Tercer bloque: \\ Experiencias subjetivas de aprendizaje}
P3.1. ¿Cuánto aprendes en esta actividad?
P3.2. ¿Es importante lo que aprendes en esta actividad?
P3.3. ¿Aprendes cosas que después te son útiles para la escuela (en esta actividad)?
P3.4. ¿Aprendes cosas que no sueles aprender en la escuela (en esta actividad)?
P3.5. ¿Aprendes cosas que te pueden ayudar en un futuro cuando seas mayor (en esta actividad)?
P3.6. ¿Aprendes cosas que te gustan y/o te interesan (en esta actividad)?
P3.7. ¿Aprendes cosas que te gustaría hacer cuando seas mayor (en esta actividad)?
P3.8. ¿Aprendes cómo comportarte cuando estás con tus amigos (en esta actividad)?
P3.9. ¿Aprendes cómo comportarte cuando estás con adultos (en esta actividad)?
P3.10. ¿Aprendes lo que está bien y lo que está mal (en esta actividad)?

\footnotetext{
Pregunta (P)

La pregunta P2.1 se presenta en cada una de las 26 actividades.

La pregunta P2.2 solo se presenta en aquellas actividades en las que el estudiante participa frecuentemente.

Las preguntas P2.3, P2.4, P2.5, P2.6, y P3.1, solo se presentan en aquellas actividades en las que el estudiante participa frecuentemente y considera importante.

La pregunta P3.2. solo se presenta en aquellas actividades en las que el estudiante participa frecuentemente, considera importante y percibe que en ella aprende cosas.

Las preguntas P3.3, P3.4, P3.5, P3.6, P3.7, P3.8, P3.9, y P3.10, solo se presentan en aquellas actividades en las que el estudiante participa frecuentemente, considera importante, percibe que aprende, y considera que aquello que ha aprendido es importante.
}

Recibido: $20-03-2020$

Aceptado: $28-05-2020$ 